\title{
Stability and asymptotic properties of a linear fractional difference equation
}

Jan Čermák*, Tomáš Kisela and Luděk Nechvátal

"Correspondence:

cermak.j@fme.vutbr.cz Institute of Mathematics, Brno

University of Technology,

Technická 2, Brno, 616 69, Czech

Republic

\begin{abstract}
This paper discusses qualitative properties of the two-term linear fractional difference equation

$$
{ }_{0} \nabla^{\alpha} y(n)=\lambda y(n),
$$

where $\alpha, \lambda \in \mathbb{R}, 0<\alpha<1, \lambda \neq 1$ and ${ }_{0} \nabla^{\alpha}$ is the $\alpha$ th order Riemann-Liouville difference operator. For this purpose, we show that this fractional equation is the Volterra equation of convolution type. This enables us to analyse its qualitative properties by use of tools standardly employed in the qualitative investigation of Volterra difference equations. As the main result, we derive a sharp condition for the asymptotic stability of the studied equation and, moreover, give a precise asymptotic description of its solutions.

MSC: 39A30; 39A12; 26A33
\end{abstract}

Keywords: fractional difference equation; Riemann-Liouville difference operator; Volterra equation; stability; asymptotic behaviour

\section{Introduction}

This paper studies stability and asymptotic properties of the linear fractional difference equation

$$
{ }_{0} \nabla^{\alpha} y(n)=\lambda y(n), \quad n=2,3, \ldots,
$$

where $0<\alpha<1, \lambda \neq 1$ are real scalars, $y(n)$ is the function defined on the set of positive integers $\mathbb{N}_{1}$, and the symbol ${ }_{0} \nabla^{\alpha}$ is the $\alpha$ th order Riemann-Liouville difference operator introduced as follows.

First, for any $v>0$, we define the $v$ th fractional sum of $y: \mathbb{N}_{1} \rightarrow \mathbb{R}$ at $n$ by

$$
{ }_{0} \nabla^{-v} y(n)=\sum_{k=1}^{n} \frac{(n-k+1)^{(v-1)}}{\Gamma(v)} y(k),
$$

where $\Gamma$ is the Euler Gamma function and

$$
(s)^{(\mu)}=\frac{\Gamma(s+\mu)}{\Gamma(s)}, \quad s \in \mathbb{N}_{1}, \mu \in \mathbb{R}
$$

\section{Springer}

(0) 2012 Čermák et al: licensee Springer. This is an Open Access article distributed under the terms of the Creative Commons Attribution License (http://creativecommons.org/licenses/by/2.0), which permits unrestricted use, distribution, and reproduction in any medium, provided the original work is properly cited. 
is the discrete power function. Then, for $0<\alpha<1$, we put

$$
{ }_{0} \nabla^{\alpha} y(n)=\nabla_{0} \nabla^{\alpha-1} y(n), \quad n=2,3, \ldots
$$

where $\nabla$ is the standard backward difference operator (corresponding to $\alpha=1$ ).

Our definition of the fractional sum (1.2) originates from the discrete backward Cauchy formula

$$
\sum_{k_{m}=1}^{n} \cdots \sum_{k_{2}=1}^{k_{3}} \sum_{k_{1}=1}^{k_{2}} y\left(k_{1}\right)=\sum_{k=1}^{n} \frac{\prod_{j=1}^{m-1}(n-k+j)}{(m-1) !} y(k)
$$

for the $m$-fold sum (for more details see, e.g. [8]). We note that the definition sum (1.2) is almost identical with related introductions in [3] and [13], where, comparing with (1.2), the term corresponding to $k=0$ has been involved as well (we emphasise that the presence of this term in (1.2) has no influence on the qualitative analysis of (1.1)). We prefer the relation (1.2), omitting the term with $k=0$, because it seems to provide, among others, a more suitable framework for possible analogues between the theory of fractional sums or differences and the continuous fractional calculus. In particular, (1.1) can be viewed as a discrete counterpart to the fractional differential equation

$$
{ }_{0} \mathrm{D}^{\alpha} x(t)=\lambda x(t), \quad t>0,
$$

where ${ }_{0} \mathrm{D}^{\alpha}$ means the $\alpha$ th order Riemann-Liouville derivative operator (for its introduction, basic properties and general information about the continuous fractional calculus we refer, e.g. to [18]). It is well known that solutions $x(t)$ of (1.4) with $0<\alpha<1$ are unbounded in a right neighbourhood of the initial point $t=0$. Consequently, the introduction of a fractional sum via the relation (1.2) with $k$ varying from $k=0$ to $k=n$ particularly implies that the value of $y(0)$ has to be defined (and finite), which is not quite consistent with this continuous pattern. For the same reason, we use the notation ${ }_{0} \nabla^{\alpha}$ (as a direct discrete analogue to the standard symbol $\left.{ }_{0} \mathrm{D}^{\alpha}\right)$, although $y(n)$ is assumed to be defined on $\mathbb{N}_{1}$ only.

Many interesting results on discrete fractional calculus have been already reported (see, e.g. $[1,3-5,15]$ and $[7,8])$, but the qualitative study of fractional difference equations is just at the beginning. In particular, considering the test equation (1.1), there are only sporadic results on the asymptotic behaviour of its solutions (see [5]), and a more systematic qualitative theory is missing.

The aim of this paper is to perform stability and asymptotic analysis of the fractional difference equation (1.1). Our investigations follow up recent research performed in [19], where the authors studied the qualitative properties of (1.4) with $0<\alpha<1$. The paper is organised as follows. In Section 2, we present an alternative (equivalent) expression of (1.1) in the form of a Volterra difference equation of convolution type. Some related tools of its qualitative investigation are mentioned as well. Section 3 discusses stability properties of (1.1) by use of analysis of the corresponding Volterra equation. We formulate here, among others, a sharp condition for its asymptotic stability. Some supplementary results on the asymptotic behaviour of the solutions of (1.1) in the stable and non-stable case are presented in Section 4. While the qualitative results, stated in Section 3 and Section 4, substantially utilise the recent progress in the theory of Volterra difference equations, Section 5 discusses some backward consequences of our analysis to this theory. In particular, 
we show that this analysis enables us to discuss some open problems in the stability theory of Volterra difference equations. Some final remarks conclude the paper.

\section{Preliminaries}

Before we start the discussion on stability and asymptotic properties of the fractional difference equation (1.1), we present its alternative form which turns out to be very convenient for such investigations.

Proposition 2.1 Let $0<\alpha<1$ and $\lambda \neq 1$. Then $y(n)$ is the solution of (1.1) if and only if $x(n)=y(n+1)$ is the solution of

$$
x(n+1)=\frac{1}{1-\lambda} \sum_{j=0}^{n}(-1)^{n-j}\left(\begin{array}{c}
\alpha \\
n-j+1
\end{array}\right) x(j), \quad n=0,1, \ldots
$$

Proof Expanding the Riemann-Liouville operator in (1.1), we have for $n=2,3, \ldots$

$$
{ }_{0} \nabla^{\alpha-1} y(n)-{ }_{0} \nabla^{\alpha-1} y(n-1)=\lambda y(n) .
$$

By the definition of fractional sum,

$$
\begin{aligned}
{ }_{0} \nabla^{\alpha-1} y(n) & =\sum_{k=1}^{n} \frac{(n-k+1)^{(-\alpha)}}{\Gamma(1-\alpha)} y(k) \\
& =\sum_{k=1}^{n} \frac{\Gamma(n-k-\alpha+1)}{\Gamma(n-k+1) \Gamma(1-\alpha)} y(k) \\
& =\sum_{k=1}^{n}\left(\begin{array}{c}
n-k-\alpha \\
n-k
\end{array}\right) y(k)=\sum_{k=1}^{n}(-1)^{n-k}\left(\begin{array}{l}
\alpha-1 \\
n-k
\end{array}\right) y(k),
\end{aligned}
$$

where we have employed the well-known property

$$
\left(\begin{array}{c}
m-p-1 \\
m
\end{array}\right)=(-1)^{m}\left(\begin{array}{l}
p \\
m
\end{array}\right), \quad m \in \mathbb{N}_{1}, p \in \mathbb{R} .
$$

Consequently, (2.2) becomes

$$
\sum_{k=1}^{n}(-1)^{n-k}\left(\begin{array}{l}
\alpha-1 \\
n-k
\end{array}\right) y(k)-\sum_{k=1}^{n-1}(-1)^{n-k-1}\left(\begin{array}{c}
\alpha-1 \\
n-k-1
\end{array}\right) y(k)=\lambda y(n),
$$

i.e.

$$
y(n)+\sum_{k=1}^{n-1}(-1)^{n-k}\left(\begin{array}{l}
\alpha-1 \\
n-k
\end{array}\right) y(k)-\sum_{k=1}^{n-1}(-1)^{n-k-1}\left(\begin{array}{c}
\alpha-1 \\
n-k-1
\end{array}\right) y(k)=\lambda y(n) .
$$

From here we get

$$
(1-\lambda) y(n)=\sum_{k=1}^{n-1}(-1)^{n-k-1}\left[\left(\begin{array}{c}
\alpha-1 \\
n-k
\end{array}\right)+\left(\begin{array}{c}
\alpha-1 \\
n-k-1
\end{array}\right)\right] y(k)
$$


hence

$$
y(n)=\frac{1}{1-\lambda} \sum_{k=1}^{n-1}(-1)^{n-k-1}\left(\begin{array}{c}
\alpha \\
n-k
\end{array}\right) y(k),
$$

which, after replacing $n$ by $n+2$ and setting $x(n)=y(n+1)$, turns into the equation (2.1).

Remark 2.2 The equation (2.1) is the Volterra difference equation of convolution type

$$
x(n+1)=\sum_{j=0}^{n} a(n-j) x(j), \quad n=0,1, \ldots
$$

where

$$
a(n)=\frac{(-1)^{n}}{1-\lambda}\left(\begin{array}{c}
\alpha \\
n+1
\end{array}\right), \quad n=0,1, \ldots
$$

Remark 2.3 The assumption $\lambda \neq 1$ is essentially the regressivity condition known from the theory of time scales (see [6]). If it is not satisfied, then (1.1) admits only the identically zero solution via the starting value $y(1)=0$. If $y(1) \neq 0$, then (1.1) has no solution.

As a result of Proposition 2.1, it is enough to analyse stability and asymptotic properties of (2.1) instead of (1.1). Before doing this, we recall some relevant stability definitions for the Volterra equation (2.3). First, we mention the standard definitions of stability and asymptotic stability adapted to the linear case.

Definition 2.4 Consider (2.3) along with the initial condition $x(0)=\phi_{0}$. Then (2.3) is said to be

(i) stable if for any real $\phi_{0}$ there exists $\varepsilon>0$ such that the corresponding solution $x(n)$ of (2.3) satisfies $|x(n)|<\varepsilon$ for all $n \in \mathbb{N}_{1}$;

(ii) asymptotically stable if $x(n) \rightarrow 0$ as $n \rightarrow \infty$ for any real $\phi_{0}$.

Remark 2.5 The notion of stability and asymptotic stability for the fractional difference equation (1.1) is defined quite analogously due to Proposition 2.1.

The relevant stability results concerning (2.3) involve also the stronger notions of uniform stability and uniform asymptotic stability. Their introductions require considering an arbitrary (finite) number of initial values. The following definitions are taken from [12].

Definition 2.6 Consider (2.3) with the initial conditions $x(k)=\phi_{k}, k=0, \ldots, m, m$ being an arbitrary nonnegative integer. Then (2.3) is said to be

(i) uniformly stable if for any $\varepsilon>0$ there exists $\delta=\delta(\varepsilon)>0$ such that if $\phi_{k}$ are reals with $\left|\phi_{k}\right|<\delta, k=0, \ldots, m$, then the corresponding solution $x(n)$ of (2.3) satisfies $|x(n)|<\varepsilon$ for all $n \in \mathbb{N}_{1}, n>m$;

(ii) uniformly asymptotically stable if it is uniformly stable and if there exists $\eta>0$ such that, for any $\varepsilon>0$ there is $N=N(\varepsilon) \in \mathbb{N}_{1}$ such that if $\phi_{k}$ are reals with $\left|\phi_{k}\right|<\eta$, $k=0, \ldots, m$, then $|x(n)|<\varepsilon$ for all $n \in \mathbb{N}_{1}, n \geq m+N$. 
A very effective method of stability analysis of (2.3) is the $\mathcal{Z}$-transform method. We recall that the $\mathcal{Z}$-transform of a sequence $u(n)$, defined on the set of nonnegative integers $\mathbb{N}_{0}$, is a complex function given by

$$
\tilde{u}(z)=\mathcal{Z}(u(n))=\sum_{k=0}^{\infty} u(k) z^{-k}
$$

where $z$ is a complex number for which this series converges absolutely. It is known that $\mathcal{Z}$-transforms can be used to solve the linear Volterra convolution equation (2.3) and find its stability or asymptotic stability conditions by analysing the roots of the associated characteristic equation

$$
z-\tilde{a}(z)=0,
$$

where $\tilde{a}(z)$ is the $\mathcal{Z}$-transform of $a(n)$ (for more details we refer to [10]). We recall the result which is the most relevant for our next study (as usual, the symbol $\ell^{1}$ used here denotes the space of sequences whose series is absolutely convergent).

Theorem 2.7 ([12, Theorem 2]) The following statements are equivalent:

(i) (2.3) is uniformly asymptotically stable;

(ii) all the roots of the characteristic equation (2.5) lie inside the unit disk, i.e.

$$
z-\tilde{a}(z) \neq 0 \quad \text { for all }|z| \geq 1
$$

(iii) $x(n) \in \ell^{1}\left(\mathbb{N}_{0}\right)$ for any solution $x(n)$ of $(2.3)$.

\section{Stability analysis}

We start with the formulation of an explicit necessary and sufficient condition for the uniform asymptotic stability of the Volterra equation (2.1). Then we discuss the asymptotic stability and stability of this equation, and summarise obtained results to present the asymptotic stability condition for the original fractional difference equation (1.1).

Theorem 3.1 Let $0<\alpha<1$ and $\lambda \neq 1$. Then (2.1) is uniformly asymptotically stable if and only if

$$
\lambda<0 \text { or } \lambda>2^{\alpha} .
$$

Proof By Theorem 2.7, we have to set up the corresponding characteristic equation (2.5) and analyse the location of its roots with respect to the unit disk.

Taking the $\mathcal{Z}$-transform of (2.4) and using the binomial theorem, we get

$$
\begin{aligned}
\tilde{a}(z) & =\frac{1}{1-\lambda} \sum_{k=0}^{\infty}(-1)^{k}\left(\begin{array}{c}
\alpha \\
k+1
\end{array}\right) z^{-k}=\frac{1}{1-\lambda} \sum_{k=1}^{\infty}(-1)^{k-1}\left(\begin{array}{l}
\alpha \\
k
\end{array}\right) z^{-k+1} \\
& =-\frac{z}{1-\lambda} \sum_{k=1}^{\infty}\left(\begin{array}{l}
\alpha \\
k
\end{array}\right)\left(-\frac{1}{z}\right)^{k}=-\frac{z}{1-\lambda}\left[\left(1-\frac{1}{z}\right)^{\alpha}-1\right]
\end{aligned}
$$


for all $z \in \mathbb{C}$ with $|z| \geq 1$. Consequently, the characteristic equation (2.5) becomes

$$
z+\frac{z}{1-\lambda}\left[\left(1-\frac{1}{z}\right)^{\alpha}-1\right]=0
$$

and, applying Theorem 2.7, the equation (2.1) is uniformly asymptotically stable if and only if

$$
1+\frac{1}{1-\lambda}\left[\left(1-\frac{1}{z}\right)^{\alpha}-1\right] \neq 0 \quad \text { for all }|z| \geq 1
$$

The nonzero roots $z_{r}$ of the characteristic equation (3.2) satisfy

$$
\left(1-\frac{1}{z_{r}}\right)^{\alpha}=\lambda
$$

We analyse (3.4) with respect to $\lambda$. First, let $\lambda<0$. Then, obviously, (3.4) has no root $z_{r}$, hence the condition (3.3) is satisfied trivially. Further, let $\lambda \geq 0$ (we recall that $\lambda \neq 1$ ). Then

$$
z_{r}=\frac{1}{1-\lambda^{1 / \alpha}}
$$

is the unique nonzero (real) root of the characteristic equation (3.2). To satisfy (3.3), we have to require

$$
\lambda^{1 / \alpha}>2, \quad \text { i.e. } \quad \lambda>2^{\alpha} .
$$

The assertion is proved.

Proposition 3.2 Let $0<\alpha<1$ and $\lambda=0$. Then (2.1) is asymptotically stable.

Proof Let $\tilde{x}(z)$ be the $\mathcal{Z}$-transform of a solution $x(n)$ of (2.1), i.e.

$$
\tilde{x}(z)=\sum_{k=0}^{\infty} x(k) z^{-k}
$$

By the well-known shift property and convolution property (see, e.g. [10]),

$$
\mathcal{Z}(x(n+1))=z \tilde{x}(z)-z x(0) \quad \text { and } \quad \mathcal{Z}\left(\sum_{j=0}^{n} a(n-j) x(j)\right)=\tilde{a}(z) \tilde{x}(z)
$$

The application of the $\mathcal{Z}$-transform to both sides of (2.1) with $\lambda=0$ then yields

$$
\tilde{x}(z)=\frac{x(0)}{\left(1-\frac{1}{z}\right)^{\alpha}} .
$$

Further, by the binomial theorem,

$$
\tilde{x}(z)=x(0) \sum_{k=0}^{\infty}\left(\begin{array}{c}
-\alpha \\
k
\end{array}\right)\left(-\frac{1}{z}\right)^{k}=x(0) \sum_{k=0}^{\infty}(-1)^{k}\left(\begin{array}{c}
-\alpha \\
k
\end{array}\right) z^{-k},
$$


and comparing this relation with (3.5), we have

$$
x(n)=x(0)(-1)^{n}\left(\begin{array}{c}
-\alpha \\
n
\end{array}\right) .
$$

To conclude the proof, we recall the binomial asymptotic relation

$$
\left(\begin{array}{c}
p \\
m
\end{array}\right) \sim \frac{(-1)^{m}}{m^{p+1} \Gamma(-p)} \quad \text { as } m \rightarrow \infty, m \in \mathbb{N}_{1}, p \in \mathbb{R} \backslash \mathbb{N}_{0}
$$

(see $[16$, p.54]), where the symbol $\sim$ means the asymptotic equivalency. This implies the asymptotic property $x(n) \rightarrow 0$ as $n \rightarrow \infty$ for any initial value $x(0)$.

Proposition 3.3 Let $0<\alpha<1$ and $0<\lambda<2^{\alpha}, \lambda \neq 1$. Then (2.1) is not stable.

Proof Let $\tilde{x}(z)$ be the $\mathcal{Z}$-transform of the solution $x(n)$ of (2.1) given by (3.5). Analogously to the previous proof, we get

$$
\tilde{x}(z)=\frac{(1-\lambda) x(0)}{\left(1-\frac{1}{z}\right)^{\alpha}-\lambda} .
$$

Setting $w=1 / z$ and considering (3.5), (3.8) we can write

$$
\frac{(1-\lambda) x(0)}{(1-w)^{\alpha}-\lambda}=\sum_{k=0}^{\infty} x(k) w^{k}
$$

The function on the left-hand side of (3.9) has the (unique) pole

$$
w_{r}=1-\lambda^{1 / \alpha} \in(-1,1) .
$$

Consequently, the series on the right-hand side of (3.9) has the radius of convergence $R<1$. By the Cauchy-Hadamard theorem,

$$
\limsup _{n \rightarrow \infty} \sqrt[n]{|x(n)|}=\frac{1}{R}>1
$$

hence (2.1) is not stable.

To summarise this section, we reformulate some of its results for the fractional difference equation (1.1). Considering this equation, we are interested especially in its asymptotic stability. Proposition 2.1, Theorem 3.1 and Proposition 3.2 imply the following assertion.

Theorem 3.4 Let $0<\alpha<1$ and $\lambda \neq 1$. Then (1.1) is asymptotically stable if

$$
\lambda \leq 0 \quad \text { or } \quad \lambda>2^{\alpha} .
$$

Remark 3.5 The condition (3.10) for the asymptotic stability of (1.1) is close to be not only sufficient, but also necessary. It remains to discuss the asymptotic stability of (1.1) with $\lambda=2^{\alpha}$, which is still an open problem. 


\section{Asymptotic analysis}

In this section, we precise some of stability results derived in the previous part. In particular, we consider the asymptotic stable case, when the solutions $x(n)$ of (2.1) are tending to zero as $n \rightarrow \infty$, and describe the exact rate of their decay. An asymptotic result concerning the non-stable case will be derived as well.

First, we note that the preliminary information on the decay rate of the solutions of (2.1) follows immediately from Theorem 2.7 and Theorem 3.1.

Corollary 4.1 Let $0<\alpha<1$ and let either $\lambda<0$ or $\lambda>2^{\alpha}$. Then

$$
x(n) \in \ell^{1}\left(\mathbb{N}_{0}\right)
$$

for any solution $x(n)$ of $(2.1)$.

To obtain a precise description of asymptotics of (2.1), we employ the following general result, which is due to Appleby et al. [2]. For any finite $r>0$, the authors introduced a class $W(r)$ of real-valued weight sequences $\gamma(n)$ by the requirements

$$
\gamma(n)>0, \quad n=1,2, \ldots, \quad \lim _{n \rightarrow \infty} \frac{\gamma(n-1)}{\gamma(n)}=\frac{1}{r}, \quad \sum_{k=0}^{\infty} \gamma(k) r^{-k}<\infty
$$

and

$$
\lim _{m \rightarrow \infty}\left(\limsup _{n \rightarrow \infty} \frac{1}{\gamma(n)} \sum_{j=m}^{n-m} \gamma(n-j) \gamma(j)\right)=0 .
$$

We reformulate here the scalar version of the relevant result (originally proved for vector Volterra difference equations), which describes the asymptotics of a solution $x(n)$ of (2.3) in terms of an appropriate sequence $\gamma(n) \in W(r)$.

Theorem 4.2 ([2, Theorem 3.2]) Suppose that, for some sequence $\gamma(n) \in W(r)$, there exists the finite limit

$$
L=\lim _{n \rightarrow \infty} \frac{a(n)}{\gamma(n)}
$$

and let

$$
\sum_{k=0}^{\infty}|a(k)| r^{-k-1}<1
$$

Then the solution $x(n)$ of (2.3) satisfies

$$
\lim _{n \rightarrow \infty} \frac{x(n)}{\gamma(n)}=\frac{L x(0)}{r(1-S)^{2}}
$$

where $S=\sum_{k=0}^{\infty} a(k) r^{-k-1}$. 
This assertion turns out to be very useful in the analysis of asymptotic properties of (2.1). It implies

Corollary 4.3 Let $0<\alpha<1$ and $|1-\lambda|>1$. Then any solution $x(n)$ of (2.1) satisfies

$$
\lim _{n \rightarrow \infty} \frac{x(n)}{n^{-(1+\alpha)}}=\frac{\alpha(1-\lambda)}{\lambda^{2} \Gamma(1-\alpha)} x(0) .
$$

Proof Consider the equation (2.1), i.e. the Volterra convolution equation (2.3) with the coefficients given by (2.4). We put $r=1$ and introduce the decreasing sequence

$$
\gamma(n)=n^{-(1+\alpha)}, \quad n=1,2, \ldots
$$

As it was remarked in [2], $n^{-(1+\alpha)} \in W(1)$ provided $0<\alpha<1$. We verify the validity of assumptions of Theorem 4.2 and, in particular, specify the values of $L$ and $S$. The first calculation employs the asymptotic property (3.7). Using this, we can write

$$
L=\frac{1}{1-\lambda} \lim _{n \rightarrow \infty} \frac{(-1)^{n}\left(\begin{array}{c}
\alpha \\
n+1
\end{array}\right)}{n^{-(1+\alpha)}}=-\frac{1}{(1-\lambda) \Gamma(-\alpha)} .
$$

Discussing (4.2), we need to sum the infinite series $\sum_{k=0}^{\infty}|a(k)|$. We have

$$
\sum_{k=0}^{\infty}|a(k)|=\frac{1}{|1-\lambda|} \sum_{k=0}^{\infty}(-1)^{k}\left(\begin{array}{c}
\alpha \\
k+1
\end{array}\right)=\frac{1}{|1-\lambda|}
$$

by use of the binomial theorem. Consequently, (4.2) holds. Analogously, we get

$$
S=\sum_{k=0}^{\infty} a(k)=\frac{1}{1-\lambda} .
$$

The property (4.4) now follows from (4.3) by use of

$$
\Gamma(1-\alpha)=-\alpha \Gamma(-\alpha) .
$$

Remark 4.4 The assumptions of Corollary 4.3 do not cover the cases $\lambda=0$ and $2^{\alpha}<\lambda \leq 2$, when the equation (2.1) is asymptotically stable as well. If $2^{\alpha}<\lambda \leq 2$ then Corollary 4.1 provides at least a partial information on the behaviour of the solutions of (2.1), namely the asymptotic property (4.1). However, this property is no longer valid if $\lambda=0$, because the corresponding Volterra equation (2.1) is not uniformly asymptotically stable. Fortunately, earlier we have derived the exact form of the solutions $x(n)$ of (2.1) with $\lambda=0$ via the relation (3.6). Then, using (3.7), we can easily get the following asymptotic result.

Corollary 4.5 Let $0<\alpha<1$ and $\lambda=0$. Then

$$
\lim _{n \rightarrow \infty} \frac{x(n)}{n^{-(1-\alpha)}}=\frac{1}{\Gamma(\alpha)} x(0)
$$

for any solution $x(n)$ of $(2.1)$. 
We conclude this part by the summary of the derived asymptotic results and their reformulation for the fractional difference equation (1.1).

Corollary 4.6 Let $0<\alpha<1$ and let either $\lambda \leq 0$ or $\lambda>2$. Then

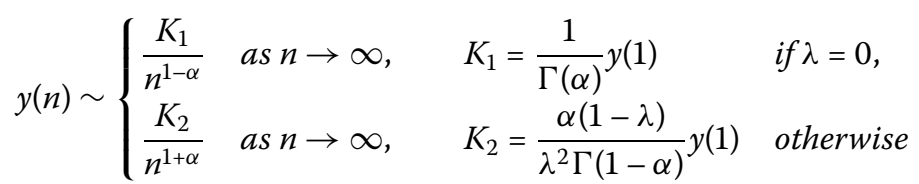

for any solution $y(n)$ of (1.1).

Now we turn our attention to the non-stable case. Recently, Atici and Eloe [5] have analysed the closed form of the solutions of (1.1) based on discrete Mittag-Leffler functions and proved that if $1 / 2 \leq \alpha<1$ and $0<\lambda<1$, then $y(n) \rightarrow \infty$ as $n \rightarrow \infty$ for any solution $y(n)$ of (1.1) with $y(1)>0$ (in our notation). We employ our approach based on the analysis of the corresponding Volterra difference equation (2.1) to obtain a slightly stronger result.

Theorem 4.7 Let $0<\alpha<1,0<\lambda<1$ and let $x(n)$ be a solution of $(2.1)$ with $x(0)>0$. Then

$$
\frac{\lambda^{1 / \alpha} x(0)}{\left(1-\lambda^{1 / \alpha}\right)^{n}}<x(n)<\frac{x(0)}{\left(1-\lambda^{1 / \alpha}\right)^{n}}, \quad n=1,2, \ldots
$$

Proof First, we introduce the function

$$
v(n)=\frac{x(0)}{\left(1-\lambda^{1 / \alpha}\right)^{n}}, \quad n \in \mathbb{Z}
$$

defining the solution of the Volterra convolution equation with infinite delay

$$
v(n+1)=\sum_{j=-\infty}^{n} a(n-j) v(j), \quad n=0,1, \ldots
$$

where

$$
a(n)=\frac{(-1)^{n}}{1-\lambda}\left(\begin{array}{c}
\alpha \\
n+1
\end{array}\right)>0, \quad n=0,1, \ldots
$$

Rewrite (4.7) as

$$
v(n+1)=\sum_{j=0}^{n} a(n-j) v(j)+g(n)
$$

where $g(n)=\sum_{j=-\infty}^{-1} a(n-j) v(j)>0$. Using the variation of constants formula (see, e.g. [12]) we have

$$
v(n)=x(n)+\frac{1}{x(0)} \sum_{j=0}^{n-1} x(n-j-1) g(j)
$$


Since $x(n)$ is positive, (4.8) implies

$$
x(n)<v(n), \quad n=1,2, \ldots,
$$

which proves the right inequality of (4.6).

Further, it follows from (4.8) that

$$
\nabla v(n)=x(n)+\left(\frac{g(0)}{x(0)}-1\right) x(n-1)+\frac{1}{x(0)} \sum_{j=1}^{n-1} x(n-j-1) \nabla g(j) .
$$

Since

$$
\nabla g(j)=\sum_{k=-\infty}^{-1} \frac{(-1)^{j-k}}{1-\lambda}\left(\begin{array}{c}
\alpha+1 \\
j-k+1
\end{array}\right) v(k)<0
$$

we get

$$
\nabla v(n)<x(n)+p x(n-1), \quad \text { where } p=\frac{g(0)}{x(0)}-1
$$

Using the binomial theorem, we can verify that

$$
p=-\frac{\lambda^{1+1 / \alpha}-(1+\alpha) \lambda^{1 / \alpha}+\alpha}{(1-\lambda)\left(1-\lambda^{1 / \alpha}\right)}
$$

We show that $p<0$, i.e.

$$
F_{\alpha}(\lambda)=\lambda^{1+1 / \alpha}-(1+\alpha) \lambda^{1 / \alpha}+\alpha>0
$$

for all $0<\alpha<1$ and $0<\lambda<1$. Indeed, we have

$$
F_{\alpha}(0)=\alpha>0, \quad F_{\alpha}(1)=0 \quad \text { and } \quad F_{\alpha}^{\prime}(\lambda)=\frac{\alpha+1}{\alpha} \lambda^{1 / \alpha}\left(1-\lambda^{-1}\right)<0
$$

for all such values of $\alpha$ and $\lambda$. Consequently, we can neglect the last term in the inequality (4.9) to obtain

$$
\nabla v(n)=\frac{\lambda^{1 / \alpha} x(0)}{\left(1-\lambda^{1 / \alpha}\right)^{n}}<x(n), \quad n=1,2, \ldots
$$

The left inequality of (4.6) is proved.

Remark 4.8 A reformulation of this asymptotic result for the fractional difference equation (1.1) is analogous as in Corollary 4.6 by use of the substitution $x(n)=y(n+1)$.

\section{Some consequences and concluding remarks}

Our stability investigation of (2.1) was based on analysis of the roots of the corresponding characteristic equation and their location with respect to the unit disk. In general, this direct approach is not practical just because of difficulties connected with the localization of 
the roots of a complex function resulting from the utilised $\mathcal{Z}$-transform method. Therefore, the following explicit criterion for the asymptotic stability of (2.3) is usually applied (for other types of stability conditions and other related asymptotic results we also refer to $[9,14]$ and [17]).

Theorem 5.1 ([10, Theorem 6.18]) Suppose that a(n) does not change sign for any $n \in \mathbb{N}_{0}$ and

$$
\left|\sum_{n=0}^{\infty} a(n)\right|<1
$$

Then (2.3) is uniformly asymptotically stable.

Till lately, it was an open question whether or not (5.1) is also necessary for the uniform asymptotic stability (or asymptotic stability) of (2.3). Only recently, Elaydi et al. [11] have constructed a class of equations (2.3) that violate the condition (5.1), but they are still asymptotically stable. In the sequel we show that (2.1) provides an alternative counterexample, which is qualitatively different from the equations considered in [11].

Doing this, we discuss the strictness of (5.1) with respect to the Volterra equation (2.1). First, notice that $\operatorname{sgn} a(n)=\operatorname{sgn}(1-\lambda)$ for all $n$, i.e. $a(n)$ does not change sign (we still assume $0<\alpha<1$ ). Furthermore,

$$
\left|\sum_{n=0}^{\infty} a(n)\right|=\frac{1}{|1-\lambda|}
$$

(see (4.5)). Hence, applying (5.1) to (2.4) we get $|1-\lambda|>1$, which is a weaker result than (3.1) yields. More precisely, if $2^{\alpha}<\lambda \leq 2$, then Theorem 3.1 implies the uniform asymptotic stability of (2.1), although (5.1) does not hold. Actually, using (2.1) we can show a little bit more, namely that the condition (5.1) is not necessary for the uniform asymptotic stability of (2.1) even if we consider it in a weaker form

$$
\left|\sum_{n=0}^{\infty} a(n)\right|<M, \quad M>0 \text { being large enough. }
$$

Example 5.2 Let $M>0$ be arbitrary. Choose $\alpha$ and $\lambda$ such that

$$
0<\alpha<1 \quad \text { and } 2^{\alpha}<\lambda<1+1 / M \text {. }
$$

Then, by Theorem 3.1, the equation (2.1) is uniformly asymptotically stable, but

$$
\left|\sum_{n=0}^{\infty} a(n)\right|=\frac{1}{\lambda-1}>M .
$$

Similarly, the fractional difference equation (1.1) rewritten into the form (2.1) may serve as an appropriate counterexample to some of other open problems posed in [10]. We can recall here the question whether or not the conditions $a(n)<0$ for all $n \in \mathbb{N}_{0}$ and $\sum_{n=0}^{\infty} a(n)<-1$ imply that $(2.3)$ is not asymptotically stable (see [10, p.299]). The answer is 
negative and follows immediately from our previous considerations on (2.1) via any choice $1<\lambda<2$ and $\alpha<\log \lambda / \log 2$.

Another specific qualitative properties of (1.1) can be observed in the seemingly simple case $\lambda=0$, when the asymptotic stability property is guaranteed for any $0<\alpha<1$ (see Proposition 3.2). This result does not agree with the limit (trivial) case $\alpha=1$, when (1.1) is stable, but not asymptotically stable. On the other hand, the qualitative behaviour of (1.1) with $\lambda=0$ is qualitatively different from the behaviour of (1.1) with other values of $\lambda$ corresponding to the asymptotically stable case $\left(\lambda<0\right.$ or $\left.\lambda>2^{\alpha}\right)$. Indeed, the decay rate of the solutions is lower than that for $\lambda<0$ and $\lambda>2^{\alpha}$ (see Corollary 4.1 and Corollary 4.6), and the asymptotic stability property for $\lambda=0$ is not uniform.

Our last remark concerns an algebraic decay of the solutions $y(n)$ of $(1.1)$ with $|1-\lambda|>1$. By Corollary 4.6, its order is equal to $1+\alpha$. It might be interesting to note that this decay rate is exactly the same as that derived in [19] for the corresponding fractional differential equation (1.4). This resemblance as well as the above derived asymptotic stability region (3.10) indicate that the fractional difference equation (1.1) could be a very suitable tool for numerical approximations of the underlying fractional differential equation. We suppose that this and other related questions will be the subject of further research.

Competing interests

The authors declare that they have no competing interests.

Authors' contributions

JČ suggested the main idea of this manuscript. TK and LN contributed by calculations, discussions and corrections. All authors read and approved the final manuscript.

\section{Acknowledgements}

The research was supported by the grant P201/11/0768 of the Czech Science Foundation and by the project FSI-S-11-3 of Brno University of Technology. The authors are grateful to the referees for their suggestions and comments which considerably helped to improve the content of this paper.

Received: 5 April 2012 Accepted: 4 July 2012 Published: 23 July 2012

References

1. Anastassiou, GA: Nabla discrete fractional calculus and nabla inequalities. Math. Comput. Model. 51(5-6), 562-571 (2010)

2. Appleby, JAD, Győri, I, Reynolds, DW: On exact convergence rates for solutions of linear systems of Volterra difference equations. J. Differ. Equ. Appl. 12(12), 1257-1275 (2006)

3. Atici, FM, Eloe, PW: Discrete fractional calculus with the nabla operator. Electron. J. Qual. Theory Differ. Equ. 2009(3), 1-12 (2009)

4. Atici, FM, Eloe, PW: Initial value problems in discrete fractional calculus. Proc. Am. Math. Soc. 137(3), 981-989 (2009)

5. Atici, FM, Eloe, PW: Linear systems of fractional nabla difference equations. Rocky Mt. J. Math. 41(2), 353-370 (2011)

6. Bohner, M, Peterson, A: Advances in Dynamic Equations on Time Scales. Birkhäuser, Boston (2003)

7. Čermák, J, Kisela, T, Nechvátal, L: Discrete Mittag-Leffler functions in linear fractional difference equations. Abstr. Appl. Anal. (2011). doi:10.1155/2011/565067

8. Čermák, J, Nechvátal, L: On (q, h)-analogue of fractional calculus. J. Nonlinear Math. Phys. 17(1), 51-68 (2010)

9. Diblík, J, Růžičková, M, Schmeidel, E: Existence of asymptotically periodic solutions of system of Volterra difference equations. J. Differ. Equ. Appl. 15(11-12), 1165-1177 (2009)

10. Elaydi, S: An Introduction to Difference Equations. Undergraduate Texts in Mathematics, 3rd edn. Springer, New York (2005)

11. Elaydi, S, Messina, E, Vecchio, A: On the asymptotic stability of linear Volterra difference equations of convolution type. J. Differ. Equ. Appl. 13(12), 1079-1084 (2007)

12. Elaydi, S, Murakami, S: Asymptotic stability versus exponential stability in linear Volterra difference equations of convolution type. J. Differ. Equ. Appl. 2(4), 401-410 (1996)

13. Gray, HL, Zhang, NF: On a new definition of the fractional difference. Math. Comput. 50(182), 513-529 (1988)

14. Kolmanovskii, VB, Castellanos-Velasco, E, Torres-Munõz, JA: A survey: stability and boundedness of Volterra difference equations. Nonlinear Anal. 53(7-8), 861-928 (2003)

15. Miller, KS, Ross, B: Fractional difference calculus. In: Proc. Int. Symp. Unival. Funct., Frac. Calc. Appl., Koriyama, Japan (1988). Ellis Horwood Ser. Math. Appl., pp. 139-152. Horwood, Chichester (1989)

16. Oldham, KB, Myland, J, Spanier, J: An Atlas of Functions, 2nd edn. Springer, New York (2008)

17. Philos, CG, Purnaras, IK: The behavior of solutions of linear Volterra difference equations with infinite delay. Comput. Math. Appl. 47(10-11), 1555-1563 (2004) 
18. Podlubny, I: Fractional Differential Equations. Academic Press, New York (1999)

19. Qian, D, Li, C, Agarwal, RP, Wong, PJY: Stability analysis of fractional differential system with Riemann-Liouville derivative. Math. Comput. Model. 52(5-6), 862-874 (2010)

doi:10.1186/1687-1847-2012-122

Cite this article as: Čermák et al.: Stability and asymptotic properties of a linear fractional difference equation. Advances in Difference Equations 2012 2012:122.

Submit your manuscript to a SpringerOpen ${ }^{\circ}$ journal and benefit from:

- Convenient online submission

- Rigorous peer review

- Immediate publication on acceptance

Open access: articles freely available online

- High visibility within the field

- Retaining the copyright to your article 